\title{
Fretting corrosion in power contacts: Electrical and thermal analysis
}

\author{
S. El Mossouess ${ }^{1,2}$, N. Benjemâa ${ }^{3}$, E. Carvou ${ }^{1}$, R. El Abdi ${ }^{1}$, L.Benmamas ${ }^{1}$, L. Doublet ${ }^{2}$ \\ ${ }^{1}$ Institut de Physique de Rennes, Université de Rennes1, Campus de Beaulieu, 35042 Rennes Cedex - France \\ ${ }^{2}$ Entreprise Valeo, Etudes Electroniques- Créteil, Europarc. 94000 Créteil -France \\ ${ }^{3}$ Entreprise Contelec- 8 rue de la Motte Baril, 35000 Rennes- France
}

Sofiane.el-mossouess@univ-rennes1.fr

\begin{abstract}
Fretting corrosion phenomenon is known as the main cause of contact resistance increasing in signal contact. But for power connectors this undesirable phenomenon in embedded systems is more complex because high current induces high voltages and subsequent Joule overheating can be expected during vibration. Our study deals with contact similar power connectors, ones submitted to vibration amplitudes up to 25 micrometres at frequencies of a few $10 \mathrm{~Hz}$ under a current ramp from 0.1 to $40 \mathrm{~A}$ under $14 \mathrm{VDC}$.

The main measured parameters are the contact voltage during vibration up to a hundred thousand cycles. The average voltage by micro-voltmeter and its fluctuations during one cycle are acquired permanently by oscilloscope.

Thermal aspects are also investigated by the use of miniature thermocouples placed as close as possible to the contact zone. It was found that a limiting voltage is reached, similar to the fritting voltage in a tarnished metal film reported by Holm. So contact resistances and the power brought by Joule effect are reduced and the temperature rise is limited. The main factors which playing a role are current, contact materials and its susceptibility to wear and debris oxidation at the interface. This behaviour is confirmed by the SEM-EDX analysis of fretting zone at different steps of fretting.
\end{abstract}

Keywords: fretting-corrosion, fritting, contact resistance, contact voltage, contact temperature, automotive connectors.

\section{INTRODUCTION}

Due to repetitive micro-displacements, fretting phenomenon was defined as an electrical and mechanical degradation of the electrical contact interface in telecommunications and more recently in power automotive connectors.

Commonly, the electrical degradation is quantified by the increase of contact resistance [1-3] deduced by contact voltage. To explain the voltage variations and nonlinearity conduction reported in the literature [4] as shown in Fig. 1, the authors focused their investigation of the effect of current and power on various regions on the rate of fretting appearance and subsequent temperature.

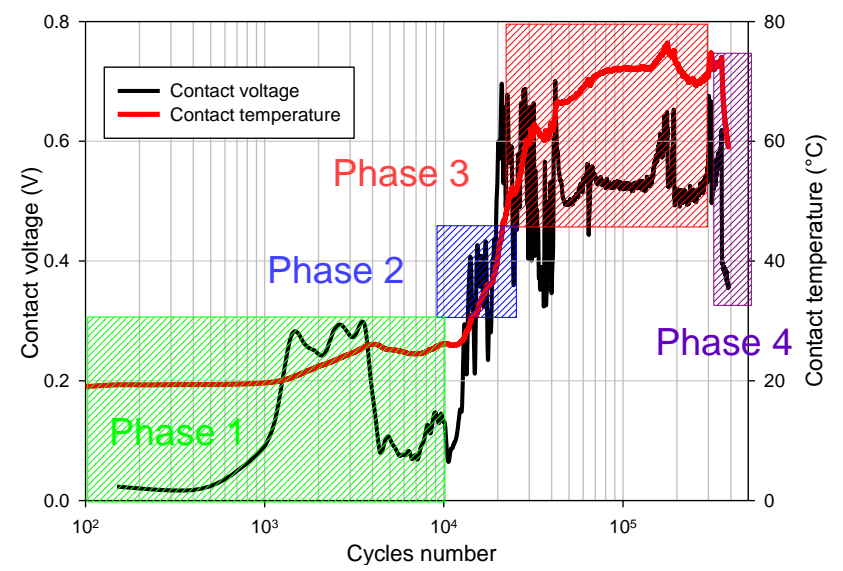

Fig.1. Evolution of contact voltage and temperature during fretting for 10A. Dashed area indicates the three phases of fretting.

Using the analysis of voltage and temperature in real time during fretting, we study the conjointly both voltage and temperature evolution. Finally, we analyse the nonlinearity and breakdown voltage of aged samples by fretting with current ramp ( 0.1 to $40 \mathrm{~A})$ in the three phases of fretting [5].

\section{EXPERIMENTAL DEVICE AND PROCEDURE}

\section{A. Sample and Vibration Characteristics}

The U-shaped samples are produced in our laboratory from cooper alloy of $0.8 \mathrm{~mm}$ thickness and coated by tin $(2 \mu \mathrm{m})$. Two contact parts are produced; the upper part with a spherical $(\mathrm{CuSn} 3 \mathrm{Zn} 9)$ shape of radius 2 and $3 \mathrm{~mm}$ and a plane part Fig. 2. In order to simulate actual operating conditions of the connectors, vibrations are imposed directly on the lower contact as shown in Fig .3. On one side the sample is firmly attached to a force sensor which is fixed to the ground while the lower part is fixed to the shaker. The vibrations are a typical sinus oscillation with a frequency of $50 \mathrm{~Hz}$ and displacement amplitude of $2 \mathrm{a}=$ $50 \mu \mathrm{m}$ p-p (peak to peak). This movement is checked and measured on the sample with a displacement sensor by laser (resolution of 0.1 microns). 


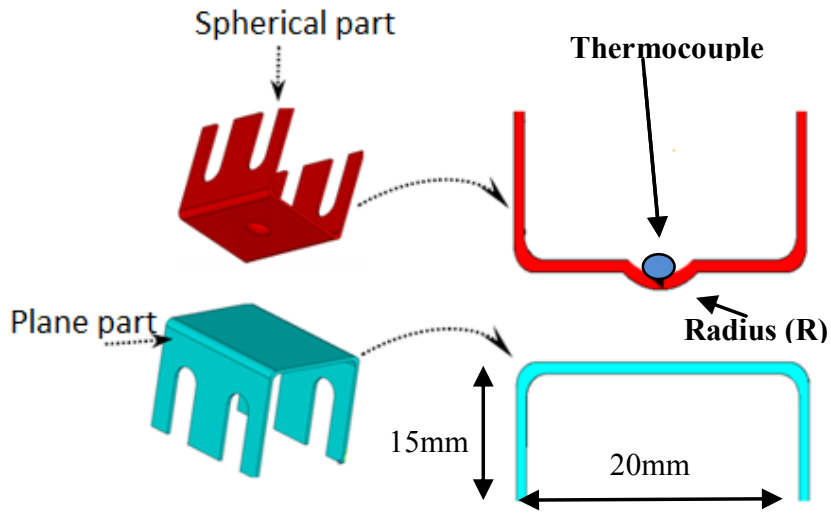

Fig.2. Sample geometry equipped with thermocouple for tests

\section{B. Experimental Procedure}

Using a micrometre indenter, a displacement is made the upper part which ensures contact with the lower at $3 \mathrm{~N}$ of contact force. The contact voltage is measured by a micro voltmeter $(0.1 \mathrm{mV}$ averaging contact voltage on one cycle). A current source with a compliance of $10 \mathrm{~V}$ supplies the sample with a current range from 1 to $40 \mathrm{~A}$. In addition, this current source has been used in the range $0.1 \mathrm{~A} 40 \mathrm{~A}$ to draw the $\mathrm{V}(\mathrm{I})$ characteristic of the contact in the different phases of wear. A miniature thermocouple was placed as close as possible to the contact zone to measure the temperature (Fig. 2) and gives a thermal effect on the electrical degradation conjointly with the contact voltage. For faster voltage fluctuations during one cycle and position in the track of displacement we use a digital oscilloscope.

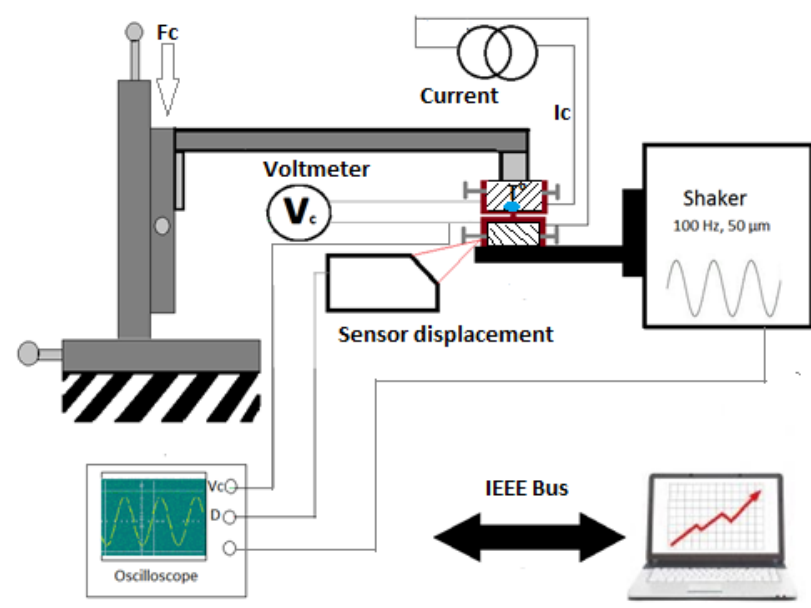

Fig.3. Fretting devices allowing displacement, voltage and temperature of contact measurements

\section{RESULTS}

The main results deal with voltage analyses during sliding in the two directions of motion and its nonlinearity in wide range of current versus aging phases as are shown in Fig. 4.

\section{A. Influence of Current on Fretting-Corrosion}

Voltage and temperature versus number of cycle are plotted for different currents levels from $1 \mathrm{~A}$ to $40 \mathrm{~A}$ and for two radius of the spherical shape. As it was expected, the saturation voltage around few hundred millivolts appears earlier when the current is increased and seems to be followed by final phase with lower and stable voltage.

Also the size of the sample acts as an accelerator factor for fretting appearance: higher radius of $3 \mathrm{~mm}$ shows a smaller number of cycles to initiate fretting and tends to higher voltage saturation than radius of $2 \mathrm{~mm}$. This is due to larger contact which could produce increasing wear and debris. Conjointly contact temperature is measured during fretting of sample at various current used in our test. We note that temperature remarkably follows the variation of contact voltage during fretting. So after the initial phase at ambient temperature it increase with some delay compared to voltage, because the thermal inertia. Similar maximum of temperature was reached at the final stage of fretting corresponding to voltage evolution. No doubt this well correlation between temperature and voltage is due to the heating by Joule effect and subsequent power $\mathrm{P}=\mathrm{Vc}$ Ic dissipated in the contact interface as related in the literature [5].
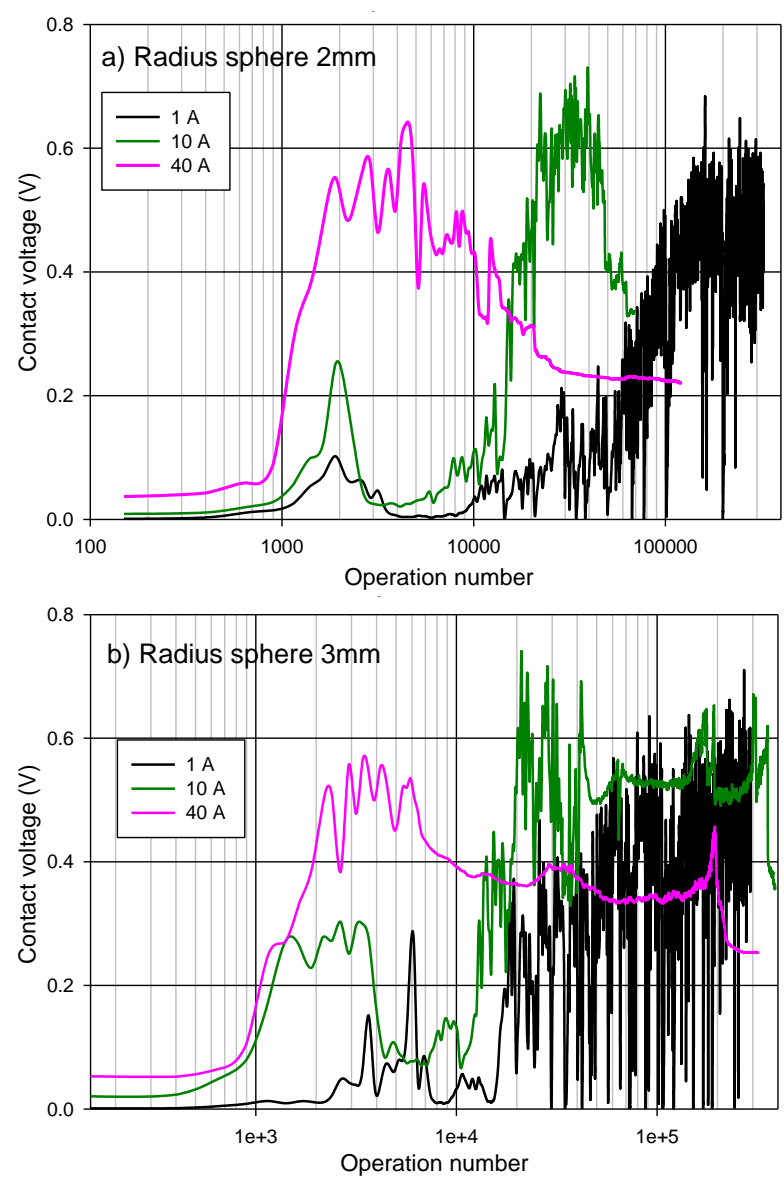

Fig.4. Contact voltage versus cycle number for three currents: a) Radius $2 \mathrm{~mm}$; b) Radius $3 \mathrm{~mm}$ 

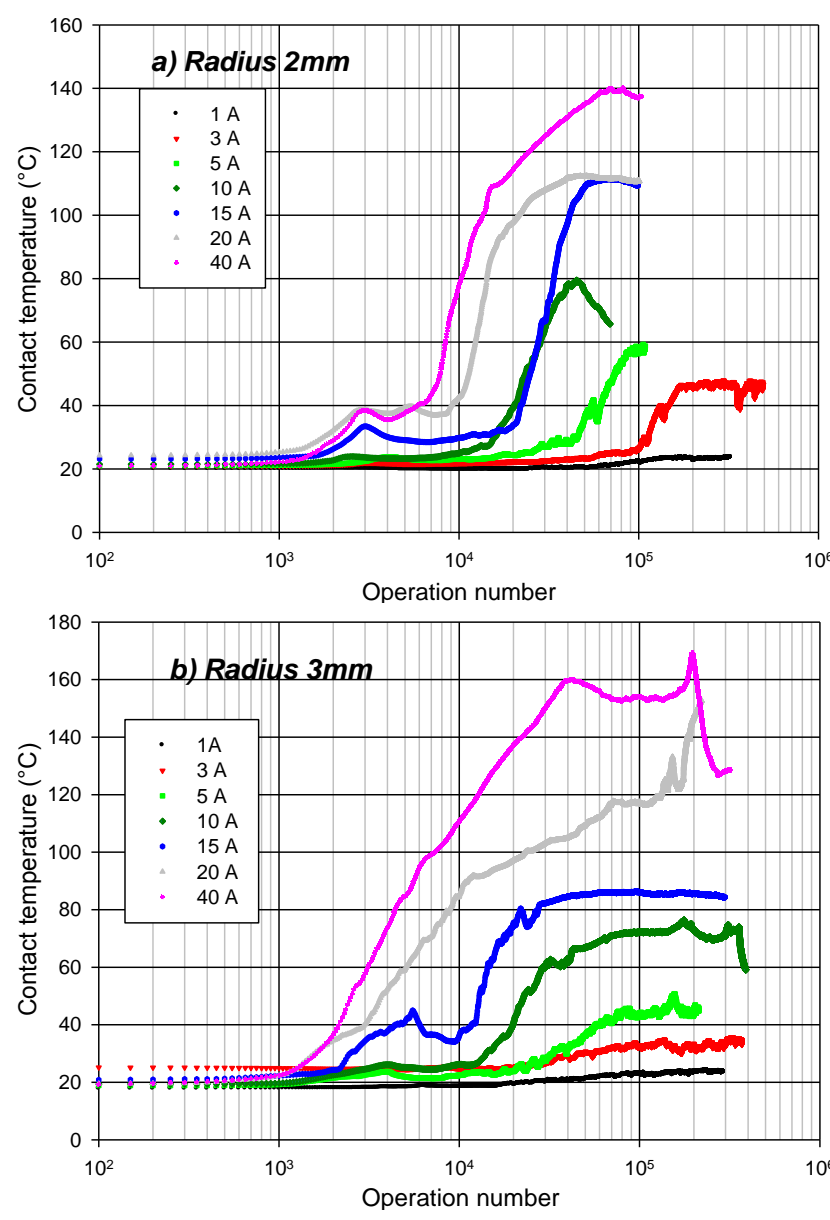

Fig.5. Contact temperature versus cycle number for three currents: a) Radius 2mm; b) Radius 3mm

As in low current level and for practical aim we calculated the number of operation needed to reach a certain value of voltage (instead of resistance) which is called as criteria of default. We can see in figure 6 the number of cycles $\mathrm{N}_{\mathrm{c}}$ needed to reach a given voltage value $(100,200,400,600 \mathrm{mV})$ in the range of current 1 to $40 \mathrm{~A}$. It appears two behaviour regions:

- In the first region dashed in left and limited to $10 \mathrm{~A}$, the relationship is $\mathrm{N}_{\mathrm{c}}=\mathrm{K}_{1} / \mathrm{I}$ where $\mathrm{K}_{\mathrm{c}}$ depend on desired voltage for example for $\mathrm{K}_{100 \mathrm{mv}}=8 \times 10^{4}$ op. $\mathrm{A}^{-1}$.

- Above 10A, the slope is increased and the relationship is $\mathrm{N}_{\mathrm{c}}=\mathrm{K}_{2} / \mathrm{I}^{2}$. This accentuation of these phenomena is due to the earlier appearance of the fretting phenomena accentuated by thermal and wear and this higher current value.

\section{B. Non-Linearity of Contact Condition}

The voltage saturation values in Fig. 4 seem to be close to a few hundred $\mathrm{mV}$ for a wide and large current domain. One can deduce that the non-linearity conduction in such interfaces is due to formation of debris and oxides.

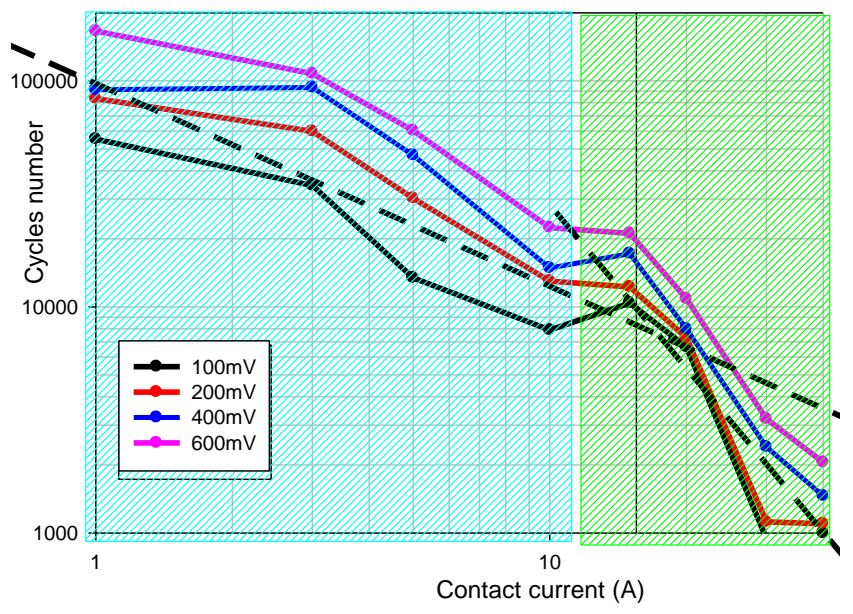

Fig.6. Cycle number versus current at different reached voltages (default criteria): dashed line indicate the power law $\left(\mathrm{N}_{\mathrm{c}}=\mathrm{K} / \mathrm{I}^{\mathrm{n}}\right)$

This phenomenon is reported in the literature [5]. As processes of oxidation of the contact surface induce poor conduction and non-linearity of current flow in the contact. For this reasons, we applied a current ramp from $0.1 \mathrm{~A}$ to $40 \mathrm{~A}$ on contact sample aged by different numbers of fretting cycle, as it is shown in Fig. 7. In the low number of cycle, corresponding to the first phase of fretting, we can remark that the contact voltage is proportional to the current. So the linearity is ensured and contact resistance remains constant $\mathrm{Rc}=\mathrm{Vc} / \mathrm{Ic}$ and close to the initial constriction value. This value can be calculated by the slope of the curve V-I. At the higher number of cycles corresponding to the second and the third phase the contact voltage follow linear evolution up to certain value of current corresponding to the power of $0.3 \mathrm{~W}$ for fretted sample at $3 \mathrm{~A}$ and $3 \mathrm{~W}$ fretted sample at $40 \mathrm{~A}$.

Also, the sample aged by fretting at $3 \mathrm{~A}$ (lower current) diverges from linearity at low current applied and is more sensitive to electrical breakdown than the fretted sample at 40A (high current). During fretting observation of the voltage fluctuation during the cycle and along the displacement track are important with many peaks with various amplitudes. This aspect is reported in our paper [6] at low electrical level. But at the final stage the contact voltage is stabilised and remain constant. 

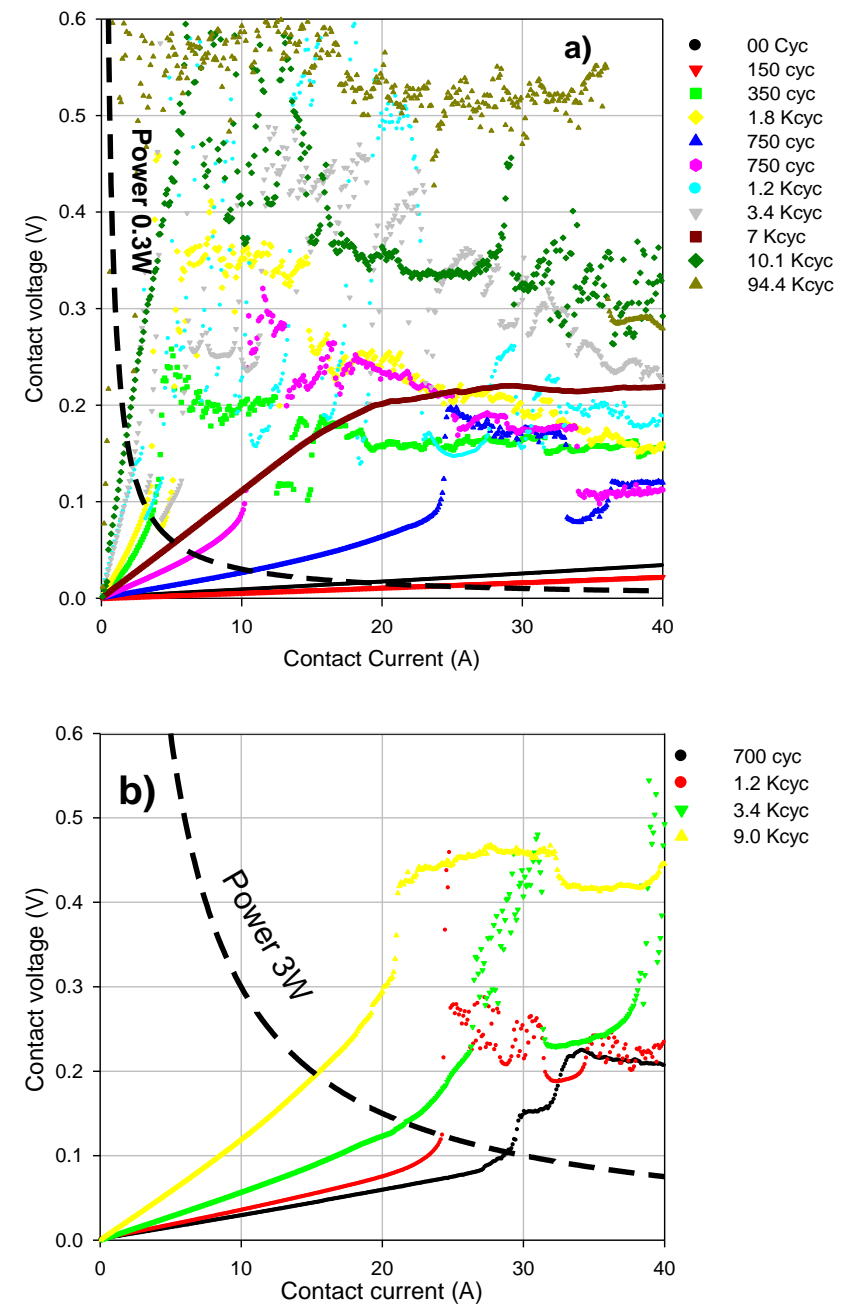

Fig.7. Contact voltage versus current for different stages of fretting: Dashed curves are constants power respectively $0.3 \mathrm{~W}$ and $3 \mathrm{~W}$ : a) Fretting at $3 \mathrm{~A}$; b) Fretting at $40 \mathrm{~A}$

\section{Contact Surface Analysis}

Regarding the evolution of voltage versus number of cycles we attempt to microscopic analysis (SEM-EDX) the elements percentage on contact surfaces at different phases of fretting. As shown in Fig. 8 the mains elements considered are the metallic coming from the subtract $(\mathrm{Zn}$, $\mathrm{Cu}$ ), coating $(\mathrm{Sn})$ and oxygen. It is clearly shown that the coating is removed progressively during the two firsts phases and disappears in the third phase where voltage is limited. In addition, during this third phase the elements of substrate appear and dominate the debris composition on the fretting surface. Finally, oxygen concentrations on the surface are increased at the first phase of fretting and remained important in the following phases two and three. We assume that the oxygen is bonded to $\mathrm{Sn}$ forming $\mathrm{SnO}_{2}$ at the initial phase, but at the final phases oxygen is bonded to cooper forming $\mathrm{Cu}_{2} \mathrm{O}$.
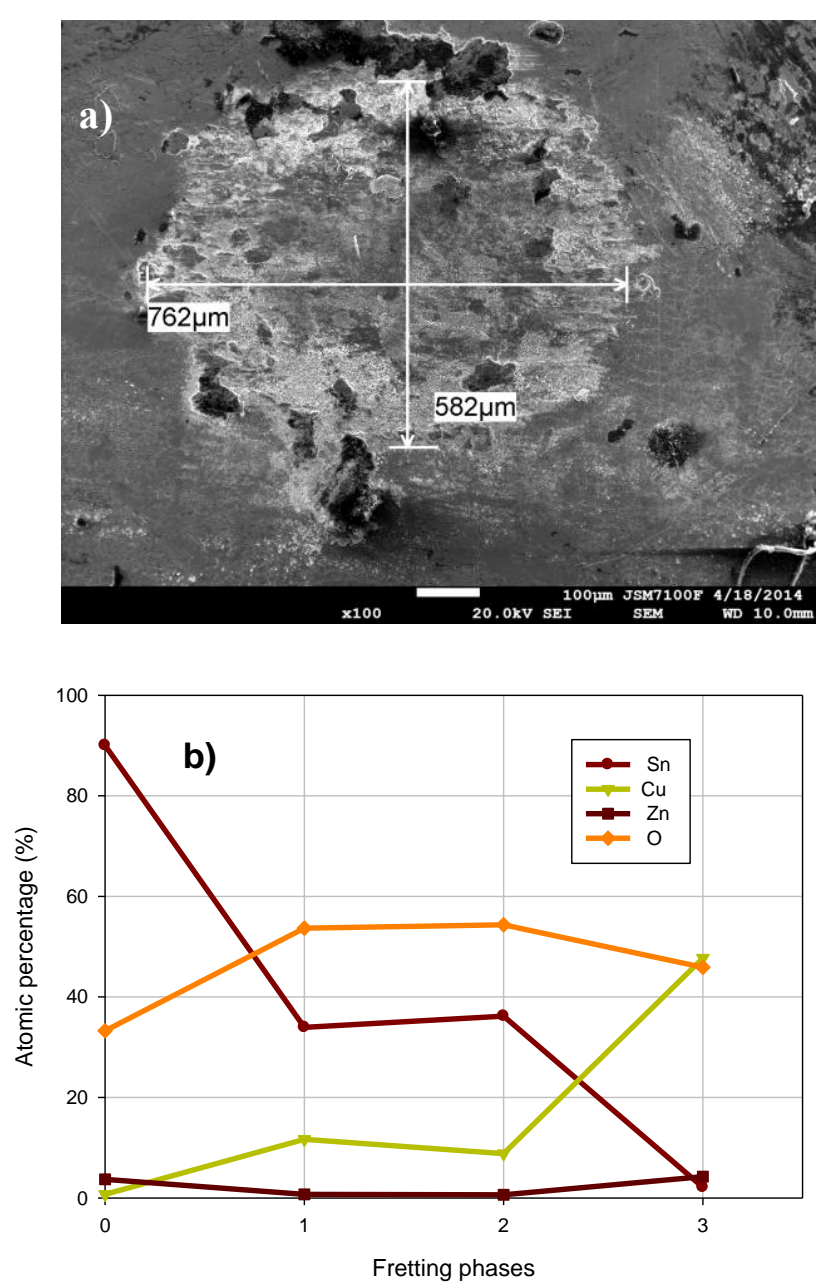

Fig.8. Fretting zone and microscopic analysis of elements $\mathrm{Sn}, \mathrm{Cu}, \mathrm{Zn}, \mathrm{O}$.

a) Fretting zone photography showing debris At the third phase, $40 \mathrm{~A}$.

b) Atomic percentage of elements at Phase 1,2 and 3 (0 is no fretted zone)

\section{CONCLUSION}

In the present study we have to expand our knowledge about the fretting phenomena to higher current and power. As in low level, the fretting evolves in the three phases but an additional final stage of stable low voltage appears in the high current, this phenomenon caused by large wear and oxidized debris in the interface. The contact temperature evolution follows similar behavior than contact voltage. Obviously, the thermal heating by Joule effect is the main cause of this over heating up to $160^{\circ} \mathrm{C}$ at $40 \mathrm{~A}$. Due to the non-linearity conduction phenomenon the default criteria of the contact should be based on the voltage level instead of contact resistance. We found that two default domains of current intensity separated by $10 \mathrm{~A}$, in the first one, the number of cycles to reach the default is proportional to the reverse current intensity. 
However this decrease is accentuated in the second region where the number of cycle is proportional to the reverse square of current. Probing this aged sample by fretting in a large current domain we found that the linearity conduction (Ohm law) is ensured at low current and limited by a power dissipation curve. The aged sample at high current (40A for example) seems to support a higher current and power before electrical breakdown by fritting voltage [7].

\section{REFERENCES}

[1] M. Antler, "Survey of contact fretting in electrical connectors» IEEE Transom components, Hybrids and Manufacturing, 1985, vol.8 pp. 87-104.

[2] J.L. Queffelec, N. Ben Jemaa and D. Travers, " Materials and contact shape studies for automobile connectors development " IEEE Trans. Comp. Hybrids, and Manuf. Technol, March 1990, Vol.14, No. 1, pp. 90-94.
[3] M. Braunovic, "Effect of fretting on the contact resistance of aluminium with different contact materials" Proceeding of the 9th ICEC Chicago, 1978, pp. 173-179.

[4] E. Carvou, N. Ben Jemaa, "Electrical contact behaviour of power connector during fretting vibration", Proceeding of Holm conf. 2007, pp.211-215.

[5] R.S. Timsit, "Electrical contact resistance properties of stationary interfaces", IEEE Holm Conference, 1988, pp.1-19.

[6] S. El Mossouess, E. Carvou, R. El Abdi, N. Benjemâa, H. Obame, L. Doublet and T. Rodari, "Analysis of temporal and spatial contact voltage fluctuation during fretting in automotive connectors", Submitted to ICEC 2014, Dresden, Germany.

[7] R. Holm Electrical "Contact, theory and applications”, 1967 Fourth edition, Springer, pp. 87. 